\title{
ULTRASONS LASER POUR LA DÉTECTION de défauts sur pièces de fabrication additive métallique
}

\author{
Célia MILLON \\ Arnaud VANHOYE \\ Anne-Françoise OBATON \\ Commissariat à l'Énergie \\ Atomique, Saclay, France \\ ${ }^{2}$ Laboratoire National \\ de Métrologie et d'Essais, \\ Paris, France \\ celia.millon@cea.fr \\ anne-francoise.obaton@lne.fr
}

La fabrication additive (FA), notamment la FA de pièces métalliques, connait un essor dans les secteurs de pointe comme l'aéronautique ou le médical de par les possibilités accrues en termes de complexité géométrique, de fonctionnalités ou encore de personnalisation des pièces. Cependant, les poudres métalliques et la fusion laser mis en œuvre dans certains procédés lors de la fabrication conduisent parfois à des défauts, comme par exemple des manques de fusion. Pour réduire les coûts de production engendrés par des pièces finies mais non conformes, la fabrication de ces pièces appelle à développer un contrôle en ligne. Les ultrasons laser (UL), non destructifs et sans contact, sont une piste prometteuse : ils combinent la sensibilité d'un contrôle par ultrasons avec la flexibilité d'un système optique.

La FA connait un essor dans les secteurs de pointe, tel que l'aéronautique ou le médical [1], de par les possibilités nouvelles qu'elle offre par rapport aux procédés classiques. Si la FA connait un réel engouement, elle n'en est pas pour autant une technologie mature et présente un certain nombre de limites. On pourra citer l'inexactitude dimensionnelle des pièces ou encore la santé matière incertaine [1-3]. Ainsi, un des axes de développement de cette technologie est le contrôle de la reproductibilité et de la robustesse jetée par une buse (figure 1).

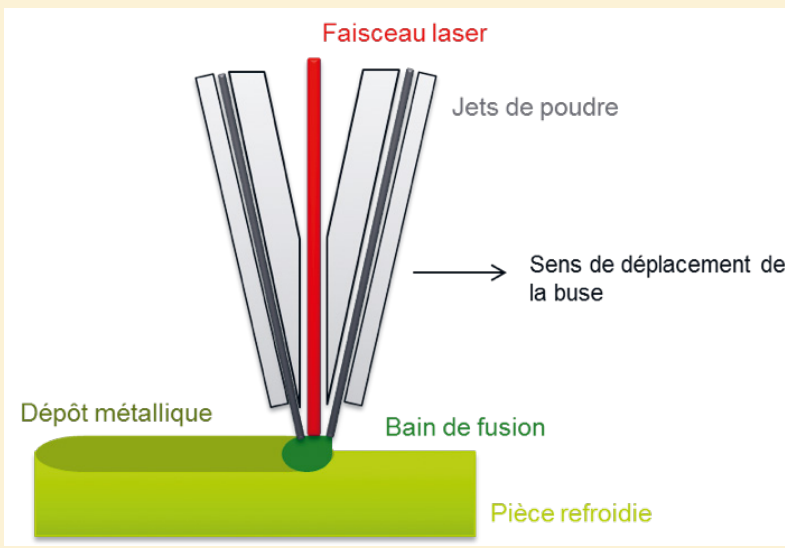

Figure 1. Schéma de principe de la catégorie de procédés de dépôt de matière sous énergie concentrée. des pièces afin de prédire l'apparition de non-conformités.

Le contrôle en cours de fabrication, appelé contrôle en ligne ou contrôle in situ, assure un suivi de la pièce au fur et à mesure de sa construction. Il permet également d'envisager une boucle de rétroaction qui arrêterait le procédé dès la détection d'une non-conformité. On comprend alors aisément l'économie possible aussi bien en temps machine qu'en matière première. Ainsi, on assiste au développement du monitoring en ligne [4]. Les ultrasons laser sont une technique à l'étude dans ce domaine [5] car ils présentent l'avantage d'être sans contact.

\section{Principe des ullitasons laser}

Les ultrasons laser (UL) sont une méthode de contrôle ultrasonore (US) sans contact. Ils peuvent donc être utilisés pour un contrôle en ligne puisqu'ils ne perturbent pas le procédé de fabrication et peuvent s'utiliser sous des contraintes de température. 


\section{Du Composant au Système}

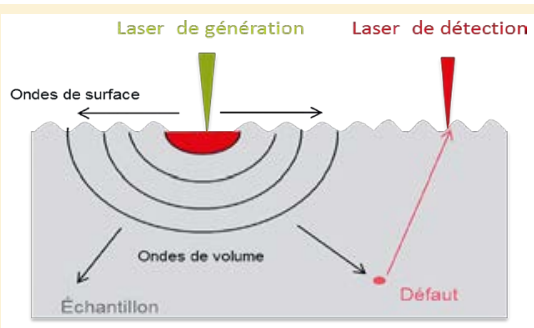

Figure 2. Principe des ultrasons laser.

Le processus de génération ultrasonore par une source laser impulsionnelle (laser de génération) est fondé sur l'interaction du faisceau lumineux avec le matériau [6]. Une brève impulsion laser échauffe la partie supérieure du matériau et entraîne localement une déformation : il y a alors création d'ondes élastiques surfaciques et volumiques dans la matière. L'interaction de ces ondes avec les défauts ou les bords de la pièce étudiée génère des déplacements de la surface. Ces déplacements sont détectés par un second laser (laser de détection) couplé à un interféromètre. La figure 2 résume le principe du contrôle par ultrasons laser.

Cette technique permet à la fois un contrôle surfacique et subsurfacique et élargit le périmètre de contrôle des techniques in situ déjà existantes [4]. Dans le cadre de notre application, on recherchera notamment à détecter les porosités et manques de fusion apparaissant en surface après le bain de fusion (interaction laser-matière) en utilisant les ondes surfaciques et plus spécifiquement les ondes dites de Rayleigh.

\section{Dispositif expérimental}

\section{Paramètres de contrôle}

Dans le cadre de nos essais, on se place dans un régime de fonctionnement thermoélastique afin de ne pas altérer la pièce au cours de sa fabrication [7]. La taille des défauts recherchés lors de l'inspection est l'ordre de $100 \mu \mathrm{m}$. Au vu de cette taille, la longueur d'onde des ondes générées doit être de l'ordre de $0,1 \mathrm{~mm}\left(\lambda_{\text {US }} \leq d_{\text {défaut }}\right)$ ce qui correspond à des fréquences de 15 $\mathrm{MHz}$ pour les ondes de Rayleigh (ces valeurs sont calculées pour la vitesse des ondes mentionnées dans l'acier, à savoir 2900 m/s). Or, la durée des impulsions laser du système UL donne la gamme de fréquence des ondes générées ainsi pour une durée d'impulsion de 10 ns, les fréquences devraient être de l'ordre de la dizaine de $\mathrm{MHz}$ [6]. Cependant, il s'est avéré que notre système ne permettait pas de détecter des ondes au-delà de $2 \mathrm{MHz}$. Cela nous a conduit à devoir étudier la corrélation entre la taille et la forme des spots laser de génération et détection et les fréquences générées et détectées.

La figure 3 donne le taux de transmission des fréquences émises pour des ondes de Rayleigh dans l'acier pour deux tailles de spot de génération. Le point de détection est considéré ponctuel.

On constate que le taux de transmission de certaines fréquences est faible (20\%). Ces fréquences ne peuvent pas être exploitées pour l'inspection et réduisent les dimensions minimales des défauts détectables par UL. Le modèle montre un taux de transmission radicalement différent entre le spot de 2,5 $\mathrm{mm}$ de diamètre et le spot de $0,2 \mathrm{~mm}$ de diamètre. Pour un spot de $2,5 \mathrm{~mm}$ de diamètre, le taux de transmission décroit dès les basses fréquences $(2 \mathrm{MHz})$. Pour les fréquences supérieures à $2 \mathrm{MHz}$, le taux de transmission est en dessous de $15 \%$. Pour le spot de $0,2 \mathrm{~mm}$, la diminution du taux de transmission n'est significative qu'aux alentours de $15 \mathrm{MHz}$. Ainsi, pour générer des fréquences de l'ordre de $15 \mathrm{MHz}$, il faut donc un spot laser dont le diamètre est inférieur ou égal à $0,2 \mathrm{~mm}$.

Par ailleurs, les dimensions spatiales des faisceaux laser ont également un impact sur la directivité des ondes. La figure 4 met en évidence des directions privilégiées des ondes de Rayleigh pour un spot circulaire et un spot linéique.

Pour un spot circulaire, aucune direction de propagation n'est favorisée,

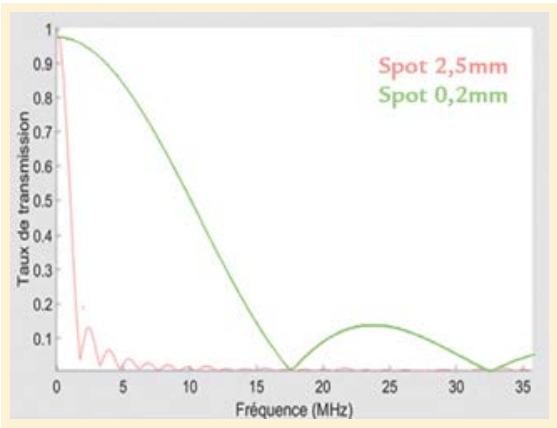

Figure 3. Réponses impulsionnelles pour des spots de génération de $0,2 \mathrm{~mm}$ (vert) et 2,5 $\mathrm{mm}$ (rose).
. Micro-positionnement

- Translation

- Rotation

- Support Miroir - etc...

. Ensembles complets
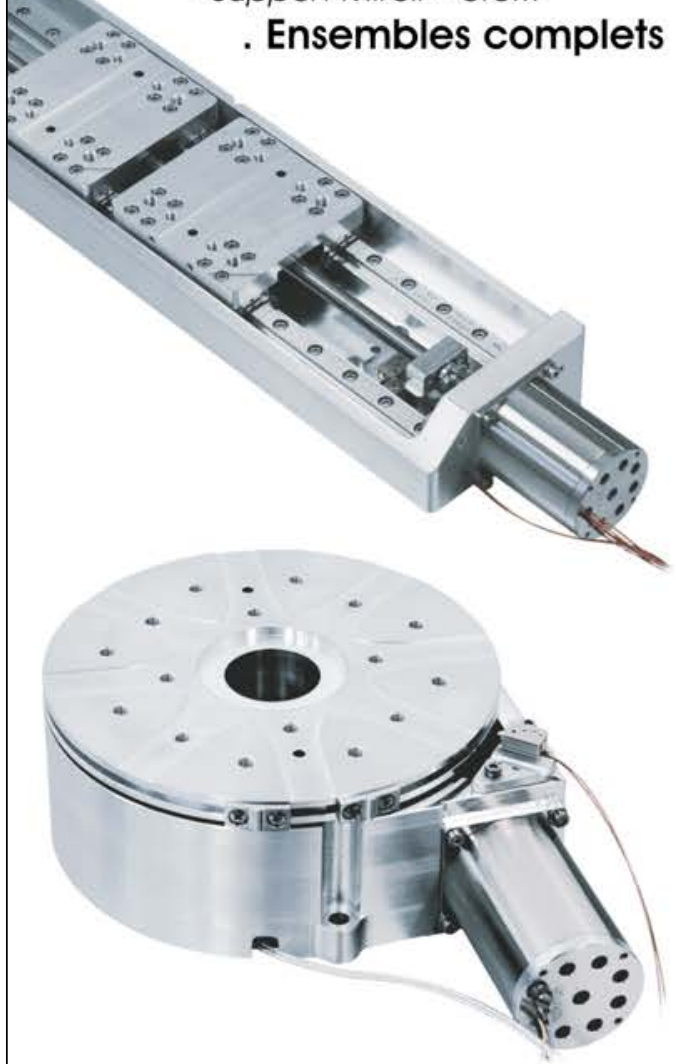

Solutions customisées et économiques

\section{Environnements : Standard / Spécifique}

Vide - Ultra-vide - Salle blanche Cryo - Hautes températures Rayonnements
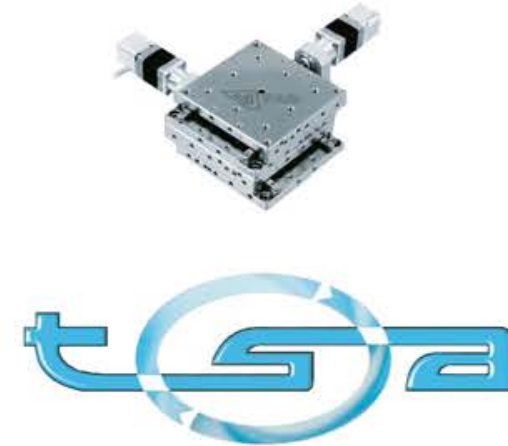

6 rue Condorcet - F-95157 Taverny Tel: +33 (0)1.30.40.81.30 


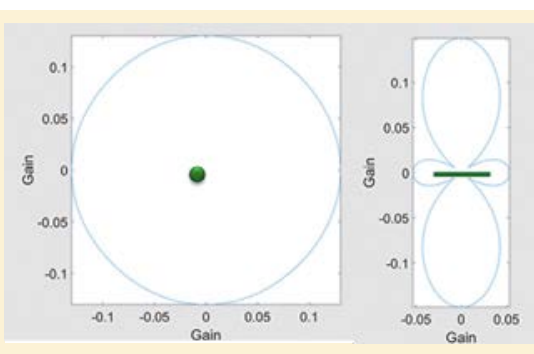

Figure 4. Diagramme de directivité des ondes de Rayleigh en régime thermoélastique pour un faisceau laser circulaire (à gauche) et un faisceau laser linéique (à droite).

Figure 5. Faisceau laser de génération focalisé sur un bloc de FA.

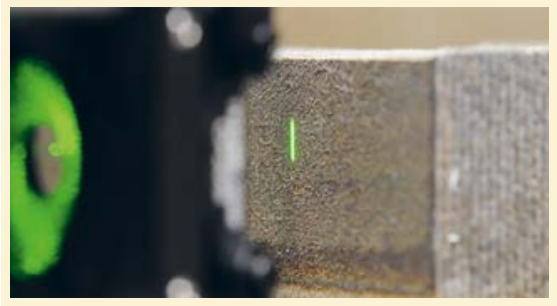

tandis que pour un spot linéique, l'aspect directionnel de la source pour les ondes de Rayleigh est démontré. La direction privilégiée des ondes de Rayleigh est perpendiculaire à la ligne laser. Ainsi, pour une détection optimale, le laser de détection doit être positionné dans cette direction (figure 5).

En conclusion, pour répondre à une configuration de contrôle optimale pour la génération d'onde de Rayleigh dans la gamme de fréquences de 0 à 15 $\mathrm{MHz}$, le système optique de focalisation du laser de génération a été adapté de sorte à permettre la réalisation d'un spot linéique de 0,2 $\mathrm{mm}$. En ce qui concerne la détection, le laser a été positionné dans la direction privilégiée avec un spot circulaire de 0,2 $\mathrm{mm}$.

\section{Dispositif d'ultrasons laser et configuration de contrôle}

Deux lasers Nd:YAG assurent la génération et la détection des ultrasons. Le laser de génération, à $532 \mathrm{~nm}$, délivre des impulsions de 7 ns pour une énergie par impulsion de $30 \mathrm{~mJ}$. Le laser de détection, à 1064 nm génère des impulsions de 80 $\mu$ s. Ce dernier est couplé à un système d'interférométrie adaptatif basé sur le mélange à deux ondes (two wave mixing, TWM) [8]. Les faisceaux laser sont injectés dans des fibres qui les acheminent vers le dispositif optique mettant en forme les faisceaux. Les deux lasers impactent la pièce sur la même face afin d'exploiter les ondes de Rayleigh. Ensuite, le système balaye la surface de l'échantillon.

\section{Pièces et défauts}

Pour comprendre l'origine des différents fronts d'onde des UL détectés et l'impact des différents paramètres de la pièce à inspecter (matériaux, géométrie de l'échantillon, dimensions des défauts, état de surface...) sur ceux-ci, nous nous sommes intéressés dans un premier temps à des défauts électro érodés sur un bloc de géométrie simple forgé et un bloc identique réalisé en FA. Les pièces inspectées sont des parallélépipèdes de $80 \times 20 \times 25 \mathrm{~mm}^{3}$ en acier $316 \mathrm{~L}$ dont les surfaces ont été polies (figure 6).

Les blocs comportent quatre entailles débouchantes usinées par électroérosion. Les entailles usinées dans ces pièces présentent différentes dimensions et visent à simuler des manques de fusion apparaissant suite à la construction d'une pièce en FA métallique. Ce sont des cas simplifiés puisque les défauts ont des bords réguliers et des dimensions calibrées. Cependant, les dimensions des défauts sont similaires à des manques de fusion induits par le procédé de FA.

\section{Résultats et discussion}

La figure 7 détaille la configuration initiale du contrôle. Les lasers sont initialement à $6 \mathrm{~mm}$ de la première entaille.

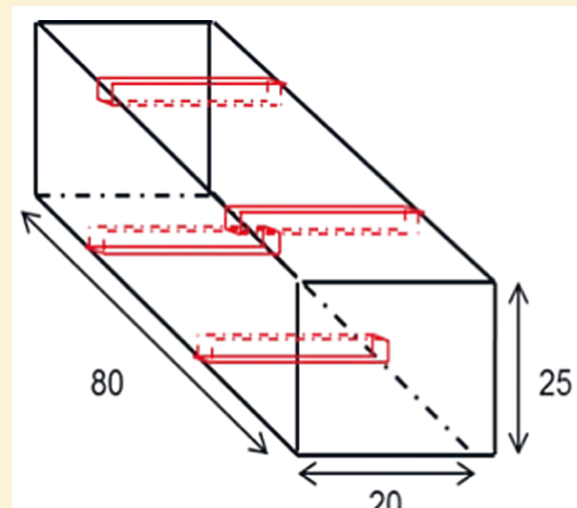

20
Le laser de génération (point vert) est situé à $3 \mathrm{~mm}$ du laser de détection (point rouge). Le balayage est matérialisé par la flèche discontinue. Le système de contrôle parcourt environ 45 $\mathrm{mm}$ par rapport à sa position initiale

La présence d'un défaut sur le trajet de l'onde perturbe le front d'onde de Rayleigh. Pour le mettre en évidence, on étudie l'amplitude de ce front d'onde en plaçant la détection à une distance permettant la séparation temporelle du front d'onde longitudinal de surface du front d'onde de Rayleigh. La surface de l'échantillon est balayée par le système de mesure de sorte que l'on suive l'évolution de la perturbation au cours du temps en fonction de la position des lasers par rapport au défaut. Les balayages permettent d'acquérir les données nécessaires aux représentations de B-scans.

La figure 7 représente schématiquement les différents fronts d'onde se propageant dans la pièce et présents sur le B-scan. Ces schémas permettent de comprendre leurs origines. Le front d'onde horizontal annoté en bleu clair correspond à l'onde de Rayleigh directe se propageant entre les deux lasers. Les fronts d'onde en pointillé bleu clair représentent les ondes de Rayleigh réfléchies par les deux entailles. Ces fronts d'onde sont la seconde signature de la présence de défauts dans la pièce. Ils sont présents sur l'ensemble des B-scans.

On note également la présence de fronts d'ondes horizontaux annotés en vert produits par les réflexions des ondes de surface avec les bords

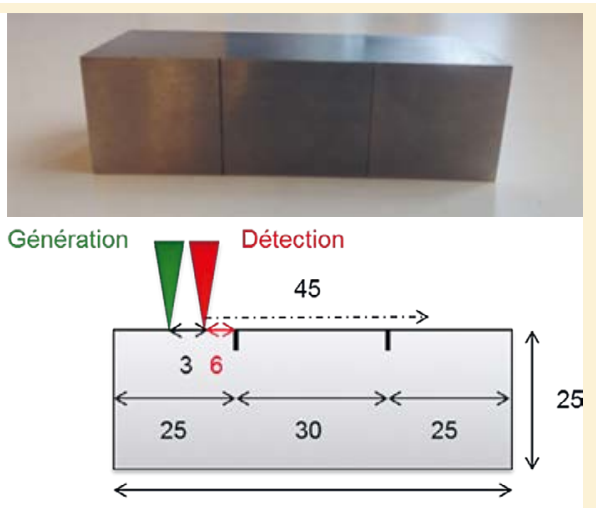

80

Figure 6. Schéma de la géométrie des pièces inspectées ; en rouge, les entailles usinées. 

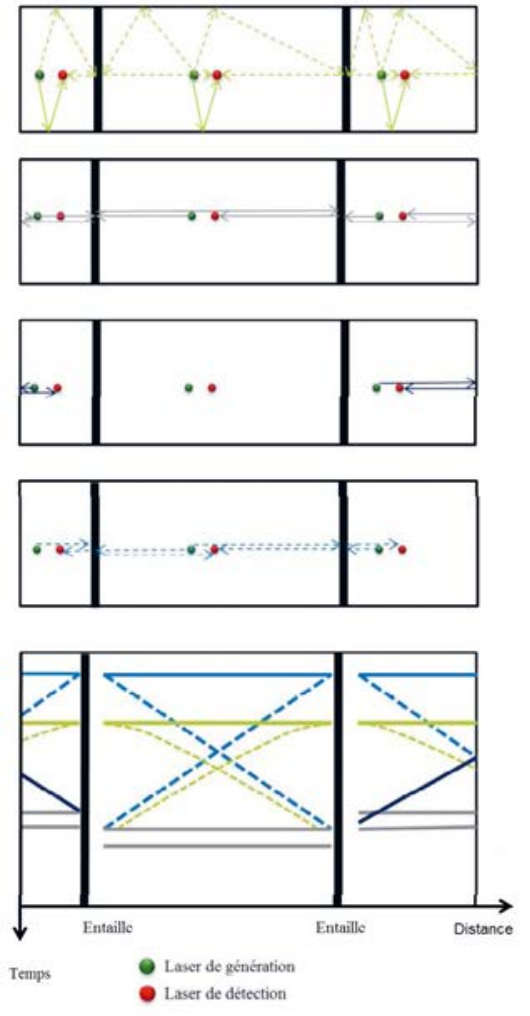

Figure 7. En haut représentation schématique des fronts d'onde d'un B-scan, en bas, schéma de la pièce et des différents parcours des ondes.

latéraux de la pièce. La réflexion de ces ondes avec l'entaille génère les fronts d'onde paraboliques vert en pointillé. Les fronts d'onde horizontaux en gris correspondent aux ondes de Rayleigh se réfléchissant entre les bords de la pièce et les entailles. Enfin, les fronts d'onde diagonaux en bleu foncé sont issus de la réflexion des ondes de surface avec les bords de la pièce. De ces observations, on peut conclure que le contrôle par ultrasons laser permet donc de détecter la présence d'entailles débouchantes dont les plus petites dimensions dans notre étude sont de $0,1 \mathrm{~mm}$ de profondeur sur 0,05 $\mathrm{mm}$ de largeur. Ce constat est fait aussi bien dans la pièce de forge que dans la pièce réalisée en FA. La limite de détection de notre système est donc au-delà de nos ambitions.

\section{Conclusion}

Ce papier a introduit l'inspection d'entailles débouchantes par ultrason lasers (UL), entailles de dimensions similaires aux défauts générés en FA. Les modèles développés, évaluant les paramètres d'influence du contrôle, ont permis de concevoir un dispositif de mesure adéquat pour la détection de défauts par les ondes de Rayleigh. Nous nous intéressons aujourd'hui également à l'impact de l'état de surface sur l'inspection par UL et nous développons des traitements d'image adaptés.

\section{POUR EN SAVOIR PLUS}

[1] J.M. Waller, B.H. Parker, K.L. Hodges, E.R. Burke, J.L. Walker, Nondestructive Evaluation of Additive Manufacturing State-of-the-Discipline Report, 2014.

[2] G. Tapia, A. Elwany, A review on process monitoring and control in metal-based additive manufacturing, J. Manuf. Sci. Eng., vol. 136, no 6, p. 060801, 2014.

[3] B.M. Sharratt, Non-destructive techniques and technologies for qualification of additive manufactured parts and processes, 2015.

[4] C. Dunsky, Process monitoring in laser additive manufacturing, Ind. Laser Solut., vol. 29, no 5, 2014.

[5] D. Cerniglia, M. Scafidi, A. Pantano, J. Rudlin, Inspection of additive-manufactured layered components, Ultrasonics, vol. 62, p. 292-298, 2015.

[6] C.B. Scruby, L.E. Drain, Laser ultrasonics techniques and applications. Adam Hilger, 1990.

[7] H. Voillaume, B. Campagne, Les ultrasons laser appliqués aux matériaux composites aéronautiques. Cofrend, 2008.

[8] R.K. Ing, J.-P. Monchalin, Broadband optical detection of ultrasound by two-wave mixing in a photorefractive crystal, Appl. Phys. Lett., vol. 59, no 25, p. 3233, 1991.

Filtres Interférentiels

De 200 á $15000 \mathrm{~nm}$

- Passe-bande

- Passe-haut

- Passe-bas

- Large bande

- Densité neutre

- Disponible en stock

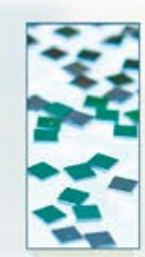

UK (parle francais): sales.uk@spectrogon.com·Tel +44 1592770000 Sweden (headquarters): sales.se@spectrogon.com·Tel +46 86382800 US: sales.us@spectrogon.com·Tel+19733311191

\section{Réseaux Holographiques}

De 150 á $2000 \mathrm{~nm}$

- Compression d'impulsion

- Télècom

- Accordabilité spectrale

- Monochromateurs

- Spectroscopie

- Disponible en stock 